\title{
Protective role of curcumin against lipopolysaccharide-induced inflammation and apoptosis in human neutrophil
}

\section{Kyu Bum Cho', Cheon Hee Park', Joungmin Kim², Tran Duc Tin ${ }^{3}$, and Sang-Hyun Kwak ${ }^{2,3}$}

Received July 30, 2019

Revised September 23, 2019

Accepted October 4, 2019

\section{Corresponding author}

Sang-Hyun Kwak, M.D., Ph.D.

Department of Anesthesiology and

Pain Medicine, Chonnam National

University Medical School and

Hospital, 42 Jebong-ro, Dong-gu,

Gwangju 61469, Korea

Tel: 82-62-220-6893

Fax: 82-62-232-6294

E-mail: shkwak@jnu.ac.kr

\section{ORCID}

https://orcid.org/0000-0001-6077-2086

This is a thesis for a Master's degree by Kyu Bum Cho.
Department of Anesthesiology and Pain Medicine, ${ }^{1}$ Kwangju Christian Hospital, ${ }^{2}$ Chonnam National University Medical School and Hospital, ${ }^{3}$ Brain Korea 21 Project, Center for Creative Biomedical Scientists at Chonnam National University, Gwangju, Korea

Background: Sepsis, an uncontrolled host response to infection, may be life-threatening organ injury. Neutrophils play a critical role in regulation of host immune response to infection. Curcumin, known as a spice and food coloring agent, possesses anti-inflammatory properties. In this study, we investigated the effects of curcumin on lipopolysaccharide (LPS)-induced neutrophil activation with its signaling pathways.

Methods: Isolated human neutrophils were incubated without or with LPS and curcumin, and the expression of pro-inflammatory cytokines, such as tumor necrosis factor alpha (TNF- $\alpha$ ), interleukin (IL)-6, and IL-8 were assessed by enzyme-linked immunosorbent assays. The expression of mitogen-activated protein kinases such as p38, extracellularsignal-regulated kinase (ERK)1/2, and c-Jun N-terminal kinase (JNK) were evaluated by Western blot analysis. Neutrophil apoptosis was also measured by fluorescenceactivated cell sorting (annexin V/propidium iodide) in LPS-stimulated neutrophils under treatment with curcumin.

Results: Curcumin attenuated expression of TNF- $\alpha$, IL- 6 , and IL-8 and the phosphorylation levels of p38 and JNK, but not ERK1/2, in LPS-stimulated neutrophils. Additionally, curcumin restored the delayed neutrophil apoptosis by LPS-stimulated neutrophils(19.7 \pm 3.2 to $38.2 \pm 0.5 \%, P<0.05$ ).

Conclusions: Our results reveal the underlying mechanism of how curcumin attenuate neutrophil activation and suggest potential clinic applications of curcumin supplementation for patients with severe sepsis and septic shock. Additional clinical studies are required to confirm these in vitro findings.

Keywords: Apoptosis; Curcumin; Inflammation; Lipopolysaccharides; Neutrophils. 
neutrophils markedly increase with prolonged survival. This process is advantageous in most infectious conditions [5]. However, over-activated neutrophils can result in massive and uncontrolled secretion of pro-inflammatory cytokines including tumor necrosis factor alpha (TNF- $\alpha$ ), interleukin (IL)6 , and IL-8 and activation of mitogen-activated protein kinase (MAPK) pathway such as p38, extracellular-signal-regulated kinase (ERK)1/2, and c-Jun N-terminal kinase (JNK). These processes may cause host tissue injury in excessive inflammatory condition [6-8]. In particular, TNF- $\alpha$, IL-6, and IL-8 are essential for the eradication of pathogens. However, the overexpression of inflammatory cytokines provokes life-threatening conditions, including sepsis and septic shock [8]. Therefore, the inhibition of pro-inflammatory cytokines, prevention of MAPKs activation, and modulation of neutrophil apoptosis should be important strategies for the treatment of excessive inflammatory diseases, such as sepsis and septic shock.

Nontoxic substances that regulate the production-mediated inflammatory responses of neutrophils may be a novel therapy in sepsis treatment; thus, many studies have attempted to identify candidate substances. Curcumin (1,7-bis-[4hydroxy-3-methoxyphenyl]-1,6-heptadiene-3,5-dione) is a yellow pigment of turmeric that is derived from the rhizome of the Curcuma longa plant and is widely used as a spice and food coloring agent. Curcumin can improve the condition in sepsis due to its anti-inflammatory, antioxidant and immunomodulatory properties. Curcumin inhibits the secretion of plasma TNF- $\alpha$ and IL-6, the activation of MAPKs, and the production of reactive oxygen species (ROS) which relate to inflammatory response $[9,10]$. Curcumin acts on MAPK p38 pathway modulating COX-2 and iNOS expression in chronic experimental colitis [11]. Curcumin also attenuates sepsisinduced acute organ dysfunction by preventing inflammation and enhancing the suppressive function of regulatory $\mathrm{T}$ cells [12]. Despite the promising evidence of the therapeutic effects of curcumin on the sepsis complication, the role of curcumin on its effect and possible mechanisms of action in human studies is not well known, in particular, the effect on human neutrophil is not documented.

The primary aim of the present study was to evaluate the roles of curcumin on neutrophil apoptosis and activation that are associated with the levels of pro-inflammatory cytokines such as TNF- $\alpha$, IL-6, and IL-8, and the expression of intracellular signaling pathways, such as the MAPK pathway.

\section{MATERIALS AND METHODS}

\section{Isolation of neutrophils}

The peripheral blood of healthy volunteers was used to isolate neutrophils under a protocol approved by the Chonnam National University Hospital Institutional Review Board (no. CNUH-2012-048). Dextran (6\%) was added and erythrocytes were sedimented under gravity for $45 \mathrm{~min}$ at room temperature. Then leukocyte-enriched pellets were collected by centrifugation at 1,100 rpm for $6 \mathrm{~min}$ and re-suspended in plateletpoor plasma. Next, leukocyte-enriched plasma was centrifuged with a plasma-Percoll gradient ( $3 \mathrm{ml} 42-51 \%$ ) at 1,100 rpm for $10 \mathrm{~min}$. Neutrophils were found at the $42-51 \%$ Percoll layer interface. Red blood cells (RBC) were removed by RBC Lysis Buffer and neutrophils were harvested by centrifugation at 3,000 rpm for $5 \mathrm{~min}$. Finally, neutrophils were re-suspended in RPMI 1640 with $10 \%$ FBS and 1\% streptomycin and penicillin (Mediatech, USA). The neutrophil viability as measured by the trypan blue exclusion test was more than $98 \%$ consistently.

\section{Cytokine analyses}

To measure the effects of curcumin on the levels of proinflammatory cytokines (TNF- $\alpha$, IL-6, and IL-8) of lipopolysaccharide (LPS)-stimulated neutrophils, isolated human neutrophils $\left(5 \times 10^{6} / \mathrm{ml}\right)$ were incubated without or with LPS (100 ng/ml, 055:B5 Escherichia coli endotoxin, Sigma-Aldrich, USA) and curcumin $(0,1,10,30,50,100 \mu \mathrm{M})$ in 24-well plates within $4 \mathrm{~h}$. Pro-inflammatory cytokines (TNF- $\alpha$, IL-6, and IL8) were measured by enzyme-linked immunosorbent assay kits (R \& D Systems, USA) according to the manufacturer's instructions.

\section{Western blot analysis}

Phosphorylation of MAPKs was determined as follows: neutrophils were lysed in protein extraction solution (PROPREPTM, Intron biotechnology, Korea) after cultured with or without LPS (100 ng/ml) and curcumin $(0,50,100 \mu \mathrm{M})$, incubated $30 \mathrm{~min}$ and then sonicated for $30 \mathrm{~s}$. Debris from the lysed cell was pelleted by centrifugation at 14,000 for 20 min and supernatant was transferred into new tube and store $-80^{\circ} \mathrm{C}$ until use. The protein concentration of each sample 
was assayed by the BCA kit (Pierce, USA), according to manufacturer's protocol.

The levels of phosphorylated and total p38 MAPK, JNK, and ERK1/2 (Cell Signaling Technologies, USA) were detected by Western blot analysis. Next, we used a chemiluminescence system and analysis software (Bio-Rad, USA) to analyse the ratio between phosphorylated and total kinase.

\section{Determination of neutrophil apoptosis by flow cytometry}

Neutrophil apoptosis was evaluated with fluorescein isothiocyanate annexin V/propidium iodide (FITC-Annexin V/ PI) according to the manufacturer's instructions (BD Bio-
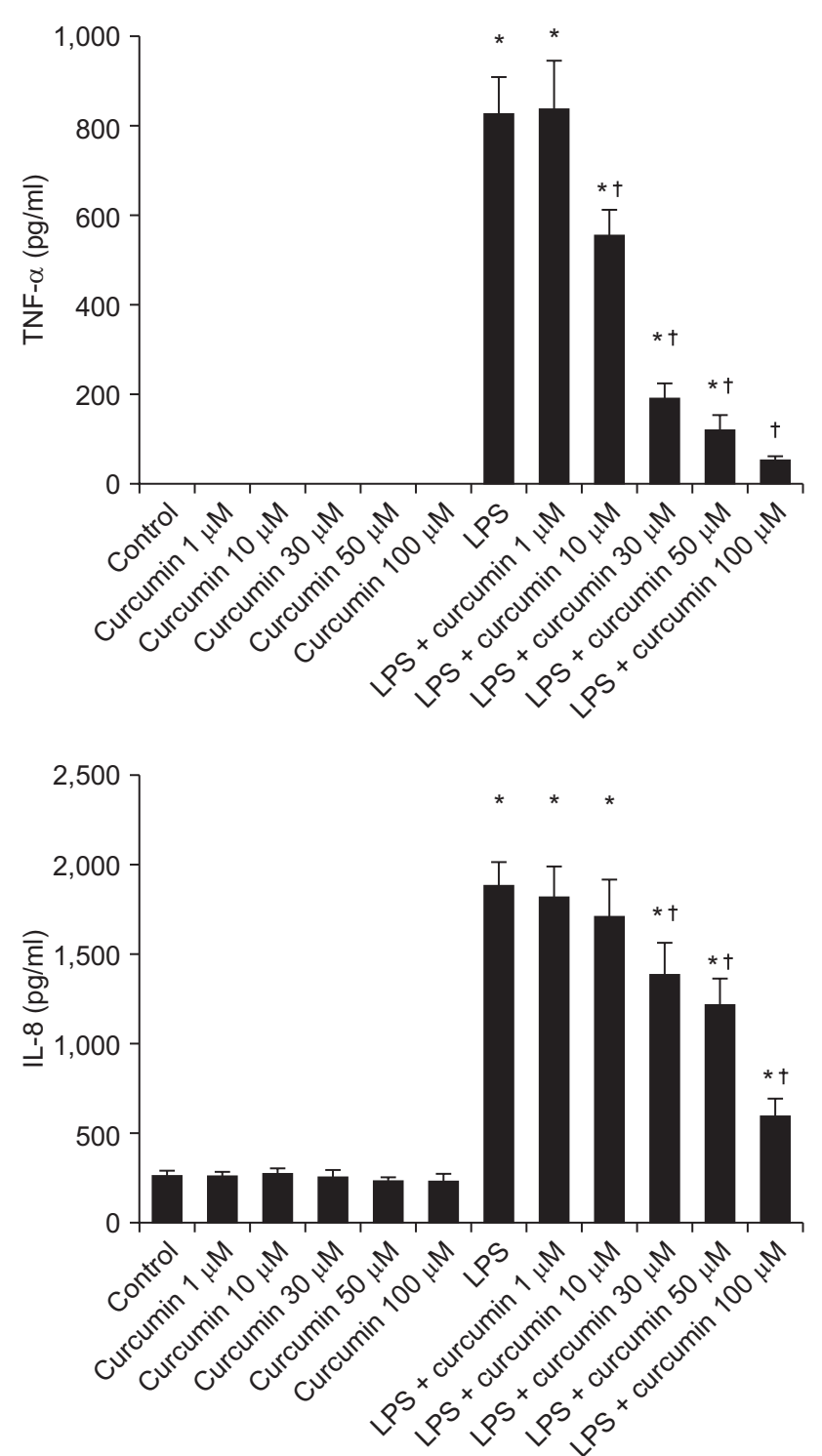

sciences, USA) with minor changes. To assess the effects of curcumin on neutrophil apoptosis, neutrophils were exposed without or with LPS (100 ng/ml) and curcumin $(100 \mu \mathrm{M})$ for $24 \mathrm{~h}$. They were washed with phosphate-buffered saline and centrifuged twice at 3,000 rpm for $5 \mathrm{~min}$, after which the cells were incubated with $300 \mu \mathrm{l}$ of binding buffer containing annexin V and PI for 15-20 min, followed by flow cytometry within $1 \mathrm{~h}$ after annexin V/PI labeling. Neutrophils in the process of apoptosis were determined by positive FITC-annexin V staining and negative PI staining.

\section{Statistical analysis}

Data are expressed as the mean \pm standard deviation for

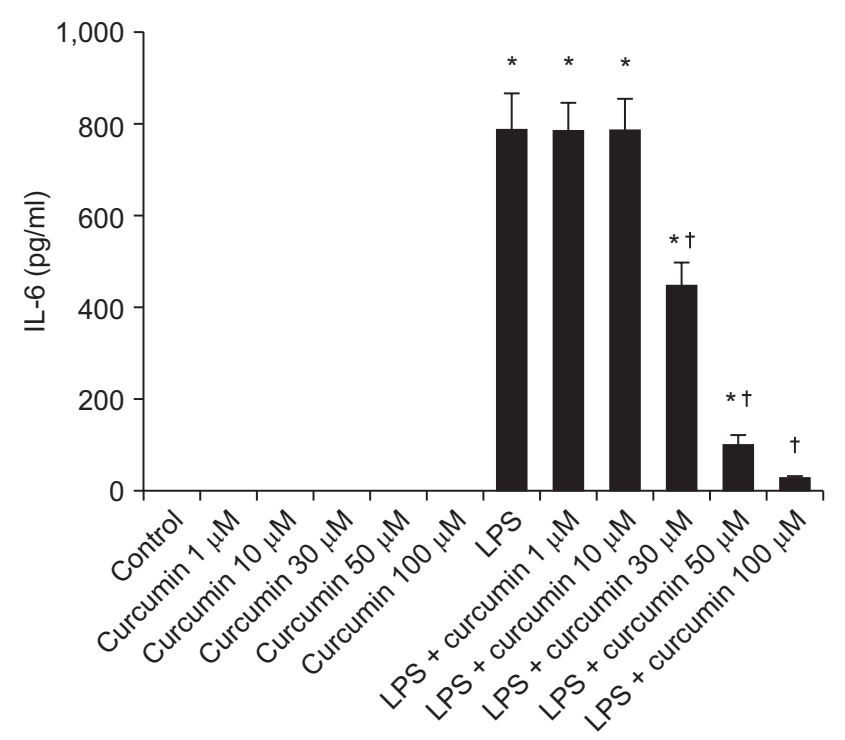

Fig. 1. The effects of curcumin on pro-inflammatory cytokine (tumor necrosis factor alpha [TNF- $\alpha$ ], interleukin [IL]-6, IL-8) expression in human neutrophil stimulated by lipopolysaccharide (LPS). Neutrophils (5 $\times 10^{6} / \mathrm{ml}$ ) from human blood were incubated for $4 \mathrm{~h}$ without or with curcumin $(1,10,30,50$, and $100 \mu \mathrm{M})$, or with LPS $(100 \mathrm{ng} / \mathrm{ml})$ or LPS plus curcumin (control, curcumin, LPS, LPS + curcumin respectively). Protein results were obtained from ELISA. Data are presented as mean $\pm \mathrm{SD}\left(\mathrm{n}=6\right.$ per group). ${ }^{*} \mathrm{P}<0.05$ vs. control. ${ }^{\dagger} \mathrm{P}<0.05$ vs. LPS. 
each group and analyzed by one-way analysis of variance followed by the Tukey-Kramer multiple comparisons test or Student's $t$-test using SPSS version 21 (IBM Co., USA). Statistical significance was defined as $\mathrm{P}<0.05$.
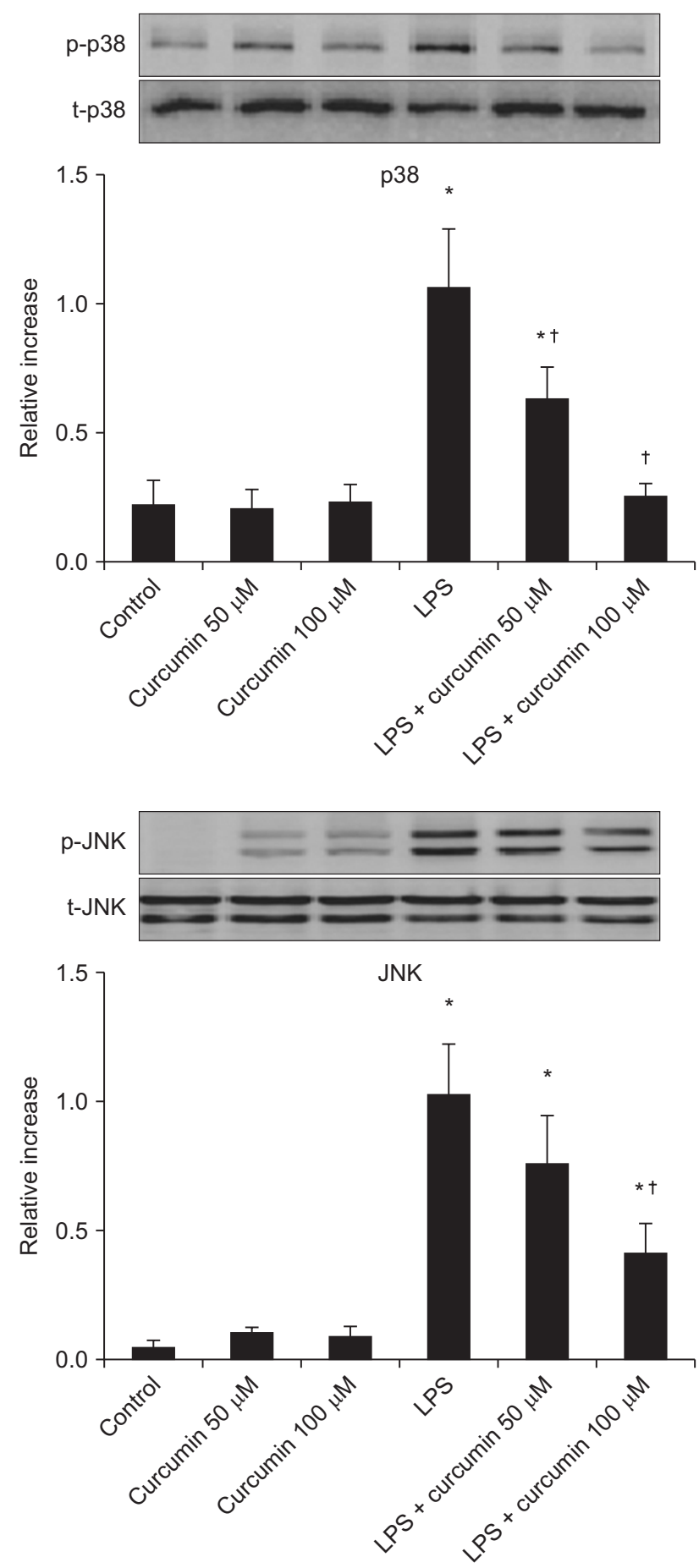

\section{RESULTS}

\section{Effects of curcumin on the cytokine expression by LPS-stimulated neutrophils}

LPS-stimulated neutrophils lead to increase inflammatory cytokines (TNF- $\alpha$, IL-6, IL-8) levels compared to those of

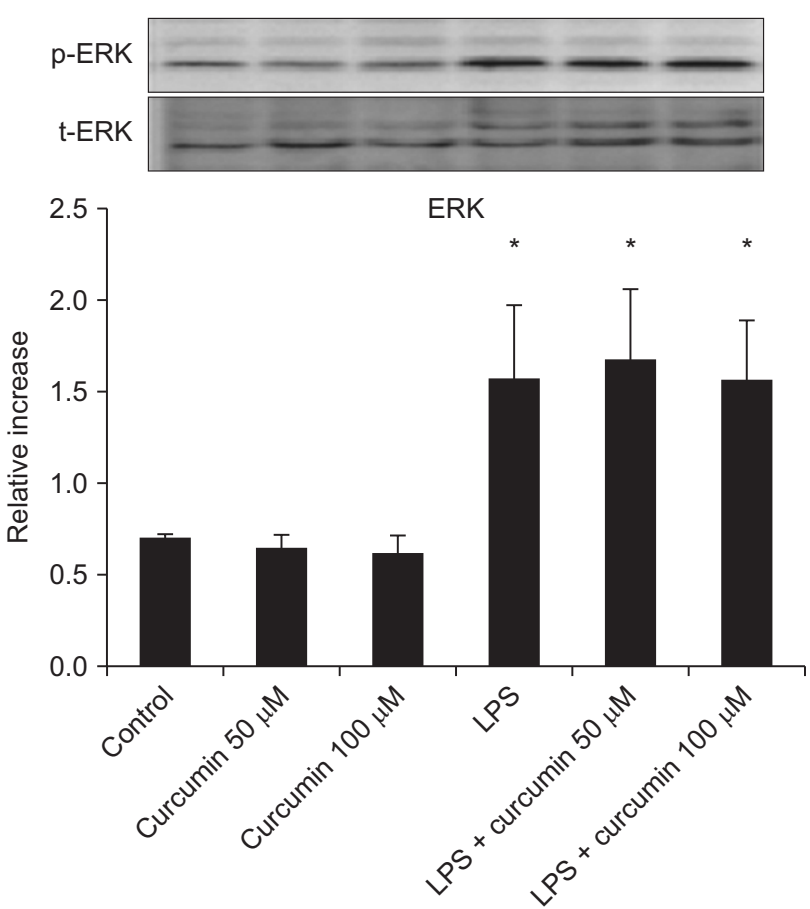

Fig. 2. The effects of curcumin on mitogen-activated protein kinases activation in human neutrophil stimulated by lipopolysaccharide (LPS). Neutrophils $\left(5 \times 10^{6} / \mathrm{ml}\right)$ from human blood were incubated for $30 \mathrm{~min}$ without or with curcumin $(50,100 \mu \mathrm{M})$ or with LPS $(100 \mathrm{ng} / \mathrm{ml})$ or LPS plus curcumin (control, curcumin, LPS, LPS + curcumin respectively). The phosphorylation $(p)$ and total $(t)$ levels of $p 38$, extracellular-signalregulated kinase (ERK)1/2, and c-Jun N-terminal kinase (JNK) were measured by Western blot analysis. Relative increase is the ratio of phosphorylated to the total kinases. Data are shown as mean $\pm \mathrm{SD}$ ( $\mathrm{n}$ $=4$ per group). ${ }^{*} \mathrm{P}<0.05$ vs. control. ${ }^{\dagger} \mathrm{P}<0.05$ vs. LPS. 
group control or curcumin alone. Curcumin attenuated this increases in dose dependently (Fig. 1). Compared to LPSstimulation alone, treatment with curcumin in LPS-stimulated neutrophils was associated with a significant reduction of TNF- $\alpha$ production at concentrations of 10, 30, 50, and 100 $\mu \mathrm{M}(824.5 \pm 87.5$ to $557.0 \pm 57.8,189.8 \pm 37.5,118.9 \pm 35.9,51.6$ $\pm 14.1 \mathrm{pg} / \mathrm{ml}$, respectively, $\mathrm{P}<0.05)$. In the same condition above, treatment with curcumin in LPS-stimulated neutrophils was associated with a significant reduction of IL-6 production at concentrations of 30,50 , and $100 \mu \mathrm{M}(784.3 \pm 85.5$ to $444.9 \pm 53.7,99.3 \pm 24.5,26.6 \pm 6.3 \mathrm{pg} / \mathrm{ml}$, respectively, $\mathrm{P}<$ 0.05). Likewise, treatment with curcumin in LPS-stimulated neutrophils was associated with a significant reduction of IL-8 production at concentrations of 30,50 , and $100 \mu \mathrm{M}(1,865.3$ \pm 141.4 to $1,367.4 \pm 197.0,1,203.8 \pm 158.1,589.8 \pm 99.3 \mathrm{pg} / \mathrm{ml}$, respectively, $\mathrm{P}<0.05)$. Curcumin alone did not increase the production of TNF- $\alpha$, IL-6, and IL-8 in human neutrophils over the range of concentrations examined.

\section{Effects of curcumin on the MAPKs activation by LPS-stimulated neutrophils}

There was a significant increase in the expression of phosphorylated p38, ERK1/2, and JNK in LPS-stimulated neu- trophils when compared to control neutrophils. Although treatment with both LPS and curcumin did not influence the ERK1/2 pathway, there was a remarkable decrease in the expression of the JNK or p38 pathways compared to LPS alone group (Fig. 2).

Compared to LPS-stimulation alone, treatment with curcumin in LPS-stimulated neutrophils was associated with a significant reduction in the ratio of phosphorylated p38 at concentrations of 50 and $100 \mu \mathrm{M}(1.06 \pm 0$ to $0.63 \pm 0.1,0.25 \pm$ 0.1 , respectively, $\mathrm{P}<0.05)$. In the same condition above, treatment with curcumin in LPS-stimulated neutrophils was associated with a significant reduction in the ratio of phosphorylated JNK at concentrations of $100 \mu \mathrm{M}(1.02 \pm 0.2$ to $0.40 \pm 0.1$, $\mathrm{P}<0.05)$. Curcumin alone did not increase the expression of phosphorylated p38, ERK1/2, and JNK in human neutrophils over the range of concentrations examined.

\section{Effects of curcumin on the apoptosis by LPS- stimulated neutrophils}

LPS stimulation inhibited neutrophil apoptosis compared to the control group ( $38.3 \pm 0.8$ to $19.7 \pm 3.2 \%, \mathrm{P}<0.05)$. Curcumin attenuated this inhibitory effects of LPS (Fig. 3). Compared to LPS-stimulation alone, treatment with curcumin in
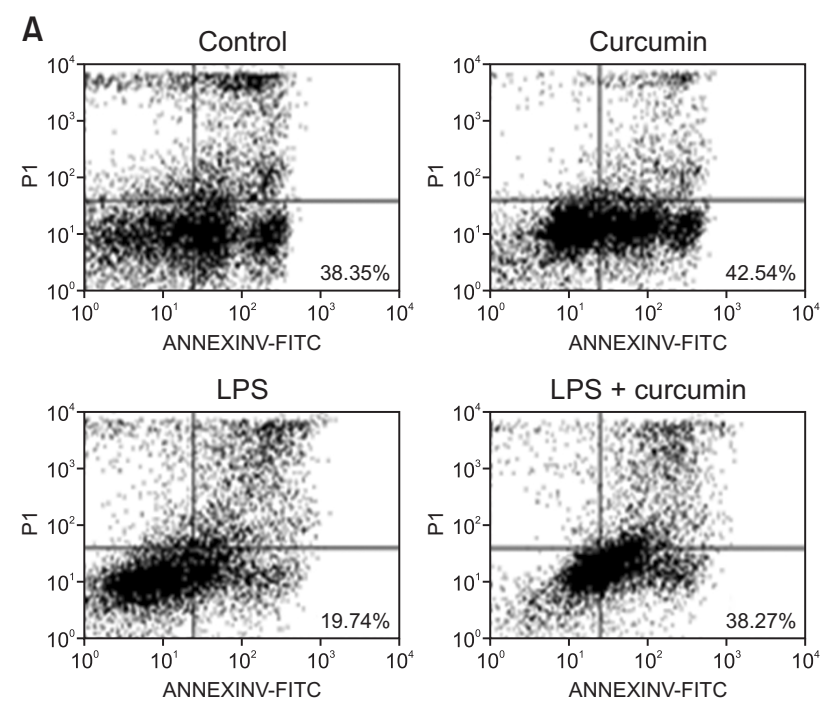

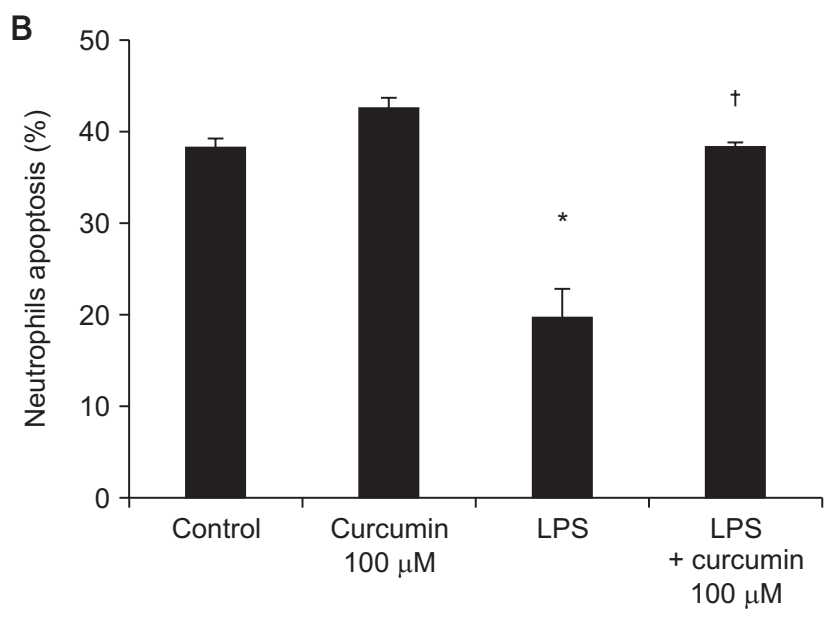

Fig. 3. The effects of curcumin on apoptosis of human neutrophils stimulated by lipopolysaccharide (LPS). Neutrophils $\left(5 \times 10^{6} / \mathrm{ml}\right)$ from human blood were incubated for $24 \mathrm{~h}$ without or with curcumin $(100 \mu \mathrm{M})$, or with LPS $(100 \mathrm{ng} / \mathrm{ml})$ or LPS plus curcumin (control, curcumin, LPS, LPS + curcumin respectively). (A) Contour diagram of fluorescein isothiocyanate annexin V/propidium iodide (FITC-Annexin V/PI) flow cytometry of neutrophils for different groups. The lower right quadrants represent the apoptotic cells, FITC-Annexin V positive and PI negative. One representative experiment out of six is shown. (B) The percentage of neutrophil apoptosis was determined for each group. Data are shown as mean \pm SD $(n=6$ per group). *P $<0.05$ vs. control. ${ }^{\dagger} \mathrm{P}<0.05$ vs. LPS. 
LPS-stimulated neutrophils was associated with a significant enhancing neutrophil apoptosis $(19.7 \pm 3.2$ to $38.2 \pm 0.5 \%, \mathrm{P}<$ 0.05). Curcumin alone did not affect neutrophil apoptosis.

\section{DISCUSSION}

The primary aim of the present study was to explore the effects of curcumin on LPS-induced neutrophil activation in terms of the stimulation of pro-inflammatory cytokines (TNF- $\alpha$, IL-6, and IL-8), the phosphorylation of MAPKs (p38, ERK1/2, and JNK), and neutrophil apoptosis. The principal finding of the present study was that curcumin play a key role in anti-inflammatory activities, such as reducing the expression of MAPKs (p38 and JNK but not ERK1/2), decreasing the level of cytokines (TNF- $\alpha$, IL-6, and IL-8), and enhancing neutrophil apoptosis by LPS-stimulated neutrophils under in vitro conditions.

LPS-induced neutrophils activate a variety of kinases associated with pro-inflammatory cytokine activation. Of these kinases, the signaling pathway induces the activation of p38, ERK1/2, and JNK which is particularly important because the decrease of MAPKs activation markedly controls the mediators of cytokine activation induced by LPS [6,7]. Under these signaling pathways, neutrophils release the numerous of oxygen radicals, proteases, leukotrienes, and pro-inflammatory cytokines $[13,14]$. Thus, inhibition of these signaling pathway may be a key strategy for the treatment of sepsis.

Inhibitors of nuclear factor kappa b (NF- $\mathrm{B})$, such as curcumin, may reduce cytokine activities. For example, pretreatment with curcumin attenuates hemorrhage/resuscitation injury-induced increases in pro-inflammatory cytokines and also inhibits the activation of NF- $\kappa \mathrm{B}$ and protein-1 [15]. Treatment with curcumin mitigates the systemic response to sepsis rats by cecal ligation and puncture. Furthermore, the intravenous administration of curcumin attenuates tissue injury, reduces death rate, and decreases the TNF- $\alpha$ protein levels [16].

The present study assessed MAPKs activity using western blot analyses because p38, ERK1/2, and JNK, which are all MAPKs, are associated with cellular damage and/or cell death $[17,18]$. These compounds are stimulated by extracellular stressors such as ROS and cytokines and can cause cell death. Moreover, MAPKs are important upstream modulators of the production of pro-inflammatory mediators and

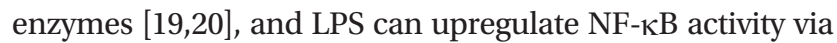
MAPK signaling [21]. In the present study, curcumin inhibited the phosphorylation levels of p38 and JNK, that were induced by LPS stimulation in neutrophils. Curcumin also likely inhibited the LPS-induced activation of p38 and JNK via alterations in $\mathrm{NF}_{-} \mathrm{K} \mathrm{B}$ signaling. Taken together, the inhibition of pro-inflammatory activities by curcumin probably occurred via pathways that converge on p38 or JNK because these kinases regulate the production of TNF- $\alpha$, IL-6, and IL-8 by LPS-stimulated neutrophils.

In this study, the rate of neutrophil apoptosis was decreased by LPS-stimulated neutrophils, whereas treatment with curcumin and LPS restored the decreased rate of neutrophil apoptosis in LPS-stimulated neutrophils. Under normal conditions, neutrophils have a short half-life (7-12 h in vivo) but exhibit an extended lifespan during sepsis. It has also been shown that several signaling pathways contribute to the resistance of neutrophils to apoptosis. For example, high levels of TNF- $\alpha$, IL-1 $\beta$, IL-6, IL-8, interferon gamma, granulocyte-colony stimulating factor, and granulocytemacrophage colony-stimulating factor in conjunction with LPS stimulation delay neutrophil apoptosis. Additionally, a prolonged neutrophil lifespan contributes to bacteria killing. However, the persistent delay of neutrophil apoptosis is involved in acute inflammatory diseases and can induce tissue injuries through their secreted products [5].

The present results are consistent with those of the above studies, because, under stimulation by LPS, they showed that neutrophils released large quantities of pro-inflammatory cytokines (TNF- $\alpha$, IL-6, IL-8) that subsequently suppressed neutrophil apoptosis. Additionally, activation of the MAPKs signaling pathways was significantly accelerated in the presence of LPS. Therefore, the inhibition of inflammatory cytokines, prevention of MAPKs activation, and modulation of neutrophil apoptosis should be important strategies for the treatment of inflammatory diseases.

In summary, the present study showed that curcumin suppressed the production of TNF- $\alpha$, IL- 6 , and IL- 8 by inhibiting P38 and JNK activation pathway and enhancing apoptosis in LPS-stimulated human neutrophils. Our results reveal the underlying mechanism of how curcumin attenuate neutrophil activation and suggest potential clinic applications of curcumin supplementation for patients with severe sepsis and septic shock. However, additional clinical studies are re- 
quired to confirm these in-vitro findings.

\section{ACKNOWLEDGEMENTS}

This study was financially supported by Chonnam National University, 2017.

\section{CONFLICTS OF INTEREST}

No potential conflict of interest relevant to this article was reported.

\section{ORCID}

Kyu Bum Cho, https://orcid.org/0000-0003-2378-5544 Cheon Hee Park, https://orcid.org/0000-0001-7986-8416 Joungmin Kim, https://orcid.org/0000-0003-1135-1968 Tran Duc Tin, https://orcid.org/0000-0003-0097-4465

\section{REFERENCES}

1. Singer M, Deutschman CS, Seymour CW, Shankar-Hari M, Annane D, Bauer M, et al. The third international consensus definitions for sepsis and septic shock (Sepsis-3). JAMA 2016; 315: 80110.

2. Ferrer R, Martin-Loeches I, Phillips G, Osborn TM, Townsend S, Dellinger RP, et al. Empiric antibiotic treatment reduces mortality in severe sepsis and septic shock from the first hour: results from a guideline-based performance improvement program. Crit Care Med 2014; 42: 1749-55.

3. Rhee C, Dantes R, Epstein L, Murphy DJ, Seymour CW, Iwashyna $\mathrm{TJ}$, et al. Incidence and trends of sepsis in US hospitals using clinical vs claims data, 2009-2014. JAMA 2017; 318: 1241-9.

4. van Zanten AR, Brinkman S, Arbous MS, Abu-Hanna A, Levy MM, de Keizer NF. Guideline bundles adherence and mortality in severe sepsis and septic shock. Crit Care Med 2014; 42: 1890-8.

5. El Kebir D, Filep JG. Targeting neutrophil apoptosis for enhancing the resolution of inflammation. Cells 2013; 2: 330-48.

6. Aomatsu K, Kato T, Fujita H, Hato F, Oshitani N, Kamata N, et al. Toll-like receptor agonists stimulate human neutrophil migration via activation of mitogen-activated protein kinases. Immunology 2008; 123: 171-80.

7. Chen L, Deng H, Cui H, Fang J, Zuo Z, Deng J, et al. Inflammatory responses and inflammation-associated diseases in organs. Oncotarget 2017; 9: 7204-18.
8. Chousterman BG, Swirski FK, Weber GF. Cytokine storm and sepsis disease pathogenesis. Semin Immunopathol 2017; 39 : 517-28.

9. Wang Y, Shan X, Dai Y, Jiang L, Chen G, Zhang Y, et al. Curcumin analog L48H37 prevents lipopolysaccharide-induced TLR4 signaling pathway activation and sepsis via targeting MD2. J Pharmacol Exp Ther 2015; 353: 539-50.

10. Wu Y, Liu Z, Wu W, Lin S, Zhang N, Wang H, et al. Effects of FM0807, a novel curcumin derivative, on lipopolysaccharideinduced inflammatory factor release via the ROS/JNK/p53 pathway in RAW264.7 cells. Biosci Rep 2018;38:BSR20180849.

11. Camacho-Barquero L, Villegas I, Sánchez-Calvo JM, Talero E, Sánchez-Fidalgo S, Motilva V, et al. Curcumin, a curcuma longa constituent, acts on MAPK p38 pathway modulating COX-2 and iNOS expression in chronic experimental colitis. Int Immunopharmacol 2007; 7: 333-42.

12. Chen L, Lu Y, Zhao L, Hu L, Qiu Q, Zhang Z, et al. Curcumin attenuates sepsis-induced acute organ dysfunction by preventing inflammation and enhancing the suppressive function of Tregs. Int Immunopharmacol 2018; 61: 1-7.

13. Alves-Filho JC, Spiller F, Cunha FQ. Neutrophil paralysis in sepsis. Shock 2010; 34 Suppl 1: 15-21.

14. Mitra S, Abraham E. Participation of superoxide in neutrophil activation and cytokine production. Biochim Biophys Acta 2006; 1762: $732-41$.

15. Gaddipati JP, Sundar SV, Calemine J, Seth P, Sidhu GS, Maheshwari RK. Differential regulation of cytokines and transcription factors in liver by curcumin following hemorrhage/resuscitation. Shock 2003; 19: 150-6.

16. Siddiqui AM, Cui X, Wu R, Dong W, Zhou M, Hu M, et al. The anti-inflammatory effect of curcumin in an experimental model of sepsis is mediated by up-regulation of peroxisome proliferatoractivated receptor-gamma. Crit Care Med 2006; 34: 1874-82.

17. Ventura JJ, Cogswell P, Flavell RA, Baldwin AS Jr, Davis RJ. JNK potentiates TNF-stimulated necrosis by increasing the production of cytotoxic reactive oxygen species. Genes Dev 2004; 18 : 2905-15.

18. Matsuzawa A, Ichijo H. Stress-responsive protein kinases in redox-regulated apoptosis signaling. Antioxid Redox Signal 2005; 7: 472-81.

19. Jung WK, Ahn YW, Lee SH, Choi YH, Kim SK, Yea SS, et al. Ecklonia cava ethanolic extracts inhibit lipopolysaccharide-induced cyclooxygenase-2 and inducible nitric oxide synthase expression in BV2 microglia via the MAP kinase and NF-kappaB pathways. Food Chem Toxicol 2009; 47: 410-7.

20. Hou RC, Chen YS, Chen CH, Chen YH, Jeng KC. Protective effect of 1,2,4-benzenetriol on LPS-induced NO production by BV2 
Anesth Pain Med Vol. 15 No. 1

microglial cells. J Biomed Sci 2006; 13: 89-99.

21. Karunakaran S, Ravindranath V. Activation of p38 MAPK in the substantia nigra leads to nuclear translocation of NF-kappaB in
MPTP-treated mice: implication in Parkinson's disease. J Neurochem 2009; 109: 1791-9. 